\section{Music Therapy}

\section{As a Treatment Modality for}

\section{Autism}

Spectrum

Disorders

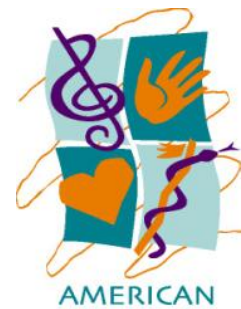

MUSIC

THERAPY

ASSOCIATION

\section{What is Music Therapy?}

Music Therapy is the clinical and evidence-based use of music interventions to accomplish individualized goals within a therapeutic relationship by a credentialed professional who has completed an approved music therapy program. It is a wellestablished allied health profession that uses music therapeutically to address behavioral, social, psychological, communicative, physical, sensorymotor, and/or cognitive functioning. Because music therapy is a powerful and non-threatening medium, unique outcomes are possible.

Music therapy may include the use of behavioral, biomedical, developmental, educational, humanistic, adaptive music instruction, and/or other models. Music therapy enhances one's quality of life, involving relationships between a qualified music therapist and individual; between one individual and another; between the individual and his/her family; and between the music and the participants. These relationships are structured and adapted through the elements of music to create a positive environment and set the occasion for successful growth.

\section{How Does Music Therapy Make a Difference for Individuals with} Diagnoses on the Autism Spectrum?
Music therapy provides a unique variety of music experiences in an intentional and developmentally appropriate manner to effect changes in behavior and facilitate development of skills.

The literature reports that most individuals with ASD respond positively to music. People with ASD often show a heightened interest and response to music, making it an excellent therapeutic tool for working with them.

Music is a very basic human response, spanning all degrees of ability/disability. Music therapists are able to meet clients at their own levels and allow them to grow from there. The malleability of music makes it a medium that can be adapted to meet the needs of each individual. Music is motivating and enjoyable. Music can promote relatedness, relaxation, learning, and self-expression. Music therapy addresses multiple developmental issues simultaneously. Music therapy can provide successoriented opportunities for achievement and mastery. The structure and sensory input inherent in music help to establish response and role expectations, positive interactions, and organization.

\section{What Do Music Therapists Do?}

Music therapists are trained professionals who accept referrals, observe clients' behavior and interactions, and assess their behavioral, emotional, psycho-social, cognitive, academic, communication, language, perceptual, sensory, motor, and musical skills.

After designing realistic goals and target objectives to address identified needs, music therapists plan and implement individualized music therapy treatment programs with strategies, procedures, and interventions to develop skills necessary to achieve an optimum level of success or quality of life for individuals with diagnoses on the autism spectrum.

Music therapists document client responses, conduct ongoing evaluations of progress and performance, and make recommendations for future consideration. Music therapists work with team members and families, providing ways to include successful music therapy techniques that support

Copyright @ 2012, American Music Therapy Association. AMTA is a 501(c)3 non-profit organization and accepts contributions which support its mission. Contributions are tax deductible as allowed by law. Rev: June 2012. 
treatment across all disciplines and in other aspects of clients' lives.

\section{Who is Qualified as a Music Therapist?}

A professional music therapist holds a bachelor's degree or higher in music therapy from one of over 70 American Music Therapy Association (AMTA) approved college and university programs. In addition to academic coursework, the bachelor's degree requires 1200 hours of clinical training, including a supervised internship. Graduate degrees in Music Therapy focus on advanced clinical practice and research.

Upon completion of the bachelor's degree, music therapists are eligible to sit for the national board certification exam to obtain the credential MT-BC (Music Therapist - Board Certified). The credential MT-BC is granted by a separate, accredited organization, the Certification Board for Music Therapists (CBMT), to identify music therapists who have demonstrated the knowledge, skills and abilities necessary to practice at the current level of the profession. For more information, contact the Certification Board for Music Therapists: www.cbmt.org

Music therapists also may be licensed, or registered, if a state licensure/registry process has been implemented by the state in which they are employed.

\section{Where Do Music Therapists Work?}

Music therapists may work with people with ASD in public school systems, where, in accordance with the Individuals with Disabilities Education Act (IDEA), music therapy is recognized as a related service that provides a "significant motivation and/or assist" in the achievement of Individual Education Plan (IEP) goals and objectives.

Music therapists also may provide service in the home, early intervention centers, Head Start programs, day care centers, specialized programs working in tandem with other professionals, day treatment centers, group homes, supportive employment sites, and at various venues within the community.

\section{Is there research to support Music Therapy services for ASD?}

Through peer-reviewed journals inside the profession such as the Journal of Music Therapy and Music Therapy Perspectives, and extensive articles in journals outside the profession, AMTA has promoted much research exploring the benefits of music therapy with individuals with diagnoses on the autism spectrum. Clinical outcomes studied have focused mainly on the use of music to address:

- Communication

- Cognition

- Behaviors (Problem//Repetitive/Stereotypic)

- Social Skills and Interaction

- Emotional Regulation

(AMTA, 2008)

The following statements represent targeted areas and rationale for using music therapy with individuals with ASD that have been the topics of published research, evidence-based practice, and/or clinical observations:

- Music holds universal appeal. It provides a bridge in a non-threatening setting between people and/or between individuals and their environment, facilitating relationships, learning, self-expression, and communication. Music captures and helps maintain attention. It is highly motivating and may be used as a natural "reinforcer" for desired responses.

- Music therapy can stimulate individuals to reduce negative and/or self-stimulatory responses and increase participation in more appropriate and socially acceptable ways. 
- Music therapy can enable those without verbal language to communicate, participate and express themselves nonverbally. Very often music therapy also assists in the development of verbal communication, speech, and language skills. The interpersonal timing and reciprocity in shared play, turn-taking, listening and responding to another person are augmented in music therapy with children and adults with autism to accommodate and address their styles of communication.

- Music therapy helps individuals with ASD identify and appropriately express their emotions.

- Because music is processed in both hemispheres of the brain, it can stimulate cognitive functioning and may be used for remediation of some speech/language skills. Recent research notes that music may engage brain regions that overlap the human mirror neuron system (Wan et al., 2010).

- Music provides concrete, multi-sensory stimulation (auditory, visual, proprioceptive, vestibular, and tactile).

- The rhythmic component of music is very organizing for the sensory systems of individuals diagnosed with autism. As a result, auditory processing and other sensory-motor, perceptual/motor, gross and fine motor skills can be enhanced through music therapy.

- Musical elements and structures provide a sense of security and familiarity in the music therapy setting, encouraging individuals with ASD to attempt new tasks in a predictable but malleable framework.
- Music therapy focuses on strengths, which in turn may be utilized to address each individual's areas of need. Many people with ASD have innate musical talents; thus, music therapy provides an opportunity for successful experiences.

For more information, please refer to AMTA's Research and Literature Pertaining to Music Therapy and Autism Spectrum Disorders.

\section{References:}

AMTA (2008). Autism Spectrum Disorders: Music Therapy Research and Evidenced-Based Practice Support. Silver Spring, MD: AMTA.

Wan, C., Demaine, K., Zipsea, L., Norton, A., \& Schlaug, G. (2010). From music making to speaking: Engaging the mirror neuron system in autism. Brain Research Bulletin 82, 161-168.

\section{How Can You Find a Music Therapist or Get More Information?}

American Music Therapy Association, Inc. 8455 Colesville Road, Suite 1000

Silver Spring, Maryland 20910, USA

Phone: (301) 589-3300 Fax: (301) 589-5175

Website: $\quad$ www.musictherapy.org

E-mail: $\quad$ info@musictherapy.org

The American Music Therapy Association (AMTA) was founded in 1998 as a result of the union of the American Association for Music Therapy, (founded in 1971), and the National Association for Music Therapy, (founded in 1950), to ensure the progressive development of the therapeutic use of music in rehabilitation, special education, and community settings. AMTA is committed to the advancement of education, training, professional standards, credentials, and research in support of the music therapy profession. 